\title{
Do Technology Shocks Drive Hours Up or Down? A Little Evidence From an Agnostic Procedure
}

\author{
Elena Pesavento \\ BARBARA ROSSI \\ EMORY UNIVERSITY \\ Duke UnIVERsity
}

October 2004

\begin{abstract}
This paper analyzes the robustness of the estimate of a positive productivity shock on hours to the presence of a possible unit root in hours. Estimations in levels or in first differences provide opposite conclusions. We rely on an agnostic procedure in which the researcher does not have to choose between a specification in levels or in first differences. We find that a positive productivity shock has a negative impact effect on hours, as in Francis and Ramey (2001), but the effect is much more short-lived, and disappears after two quarters. The effect becomes positive at business cycle frequencies, as in Christiano et al. (2003), although it is not significant.
\end{abstract}


Keywords: Technology shocks, persistence, impulse response functions, Real Business Cycle Theory.

JEL Classification: C32, C12, F40.

We thank Robert Vigfusson and Neville Francis for providing codes to replicate their results and for comments, Robert Chirinko and seminar participants at Emory, the 2004 Duke Conference on Forecasting, and the 2004 Summer Meetings of the Econometric Society for comments. We also thank Pedro Duarte and Viktor Todorov for research assistance in the early stages of the project. All mistakes are ours.

Corresponding author: Elena Pesavento, Department of Economics, Emory University, Emory GA30322, USA. Phone: (404) 712 9297. E-mail: epesave@emory.edu. 


\section{Introduction}

According to Real Business Cycles models, hours worked should rise after a positive permanent shock to technology. However, the empirical validity of this theoretical implication has been questioned in the recent literature. For example, Gali (1999) identifies technology shocks as the only shocks that have an effect on labor productivity in the long run, and estimates a persistent decline of hours in response to a positive technology shock. As Gali (1999) points out, this result is more consistent with the predictions of a New Keynesian model than those of standard Real Business Cycle models. Other papers have reached similar conclusions (see for example Shea (1999) and Francis and Ramey (2001)), which spurred a line of research aimed at developing general equilibrium models that can account for this empirical finding (see for example Uhlig (2003), Francis et al. (2003) and Gali and Rabanal (2004)).

In a recent paper, Christiano Eichenbaum and Vigfusson (2003) challenge these empirical results. Using the same identifying assumption as Gali (1999), Christiano et al. (2003) find evidence that a positive technology shock drives hours worked up, not down. It seems that the estimated effects of technology shocks crucially depend on whether the empirical analysis is specified in levels or in differences. In fact, Gali (1999), Shea (1999) and Francis and Ramey (2001) specify hours in first differences and report that hours worked fall after a positive technology shock. On the other hand, Christiano et al. (2003) use hours in levels and report that hours worked increase. In Christiano et al. (2003) words: "the difference must be due to different 
maintained assumptions. As it turns out, a key culprit is how we treat hours worked".

Whether hours worked is a stationary or an exactly integrated process is then a key assumption in the current debate on the effects of technology shocks on business cycles. However, it is practically difficult to choose between specifications in levels or in first differences on the basis of unit root tests, because of their low power. Pesavento and Rossi (2003) show that, in the presence of a root close to unity, impulse response function estimates and confidence bands that rely on unit root pretests have bad small sample properties (in terms of median unbiasedness and coverage rates). Impulse responses based on VARs estimated in levels or first differences have bad coverage properties as well, unless the true data generating process is not persistent (in which case levels are appropriate) or it has an exact unit root (in which case first differences are appropriate).

We provide empirical evidence based on an agnostic empirical estimation procedure proposed by Pesavento and Rossi (2003). The estimation is agnostic in that it does not impose either a unit root or stationarity. These authors show that their method is robust to the presence of highly persistent processes and, thus, it is appropriate if the researcher aims at analyzing the effect of technology shocks on hours worked without making assumptions on the order of integration of the series. We find that a positive productivity shock has a negative impact effect on hours worked, but this effect disappears more quickly than in Francis and Ramey (after only 2 quarters), and it becomes quickly positive. 


\section{Methodology}

Let the data generating process (hereafter DGP) be:

$$
(I-\Phi L) w_{t}=u_{t} \quad t=1,2, \ldots T
$$

where $w_{t}=\left[\begin{array}{ll}n_{t} & f_{t}\end{array}\right]^{\prime}$ is a $(2 \times 1)$ vector of variables, where $n_{t}$ is the log of per capita hours worked in the business sector and $f_{t}$ is average labor productivity. $u_{t}$ is a $(2 \times 1)$ stationary and ergodic moving average sequence:

$$
u_{t}=\Theta(L) \epsilon_{t}
$$

$\epsilon_{t}$ is a martingale difference sequence with covariance matrix $\Sigma, \Theta(L) \equiv \sum_{i=0}^{\infty} \Theta_{i} L^{i}$, $\Theta_{0}=I, I$ is the $(2 \times 2)$ identity matrix and $\Omega^{1 / 2} \equiv \Theta(1) \Sigma^{1 / 2}$ is invertible.

Note that (1) and (2) are simply another way of writing a VAR, in terms of the roots rather than in the usual linear expression with lagged endogenous variables. This representation is convenient for our purposes because it distinguishes the longrun dynamics, captured by $\Phi$, from the short-run dynamics, described by $\Theta(L)$. In fact, to allow a unit root in $f_{t}$ and high persistence in $n_{t}$, we let $\Phi=\left[\begin{array}{cc}\rho & 0 \\ 0 & 1\end{array}\right]$, where $\rho$ is close to one in a sense made precise below.

The objects of interest are the structural shocks, denoted by $\eta_{t}$, which are related 
to the VAR residuals $\epsilon_{t}$ by the following relationship:

$$
\eta_{t}=A_{0} \epsilon_{t}
$$

We let $\eta_{t}=\left[\begin{array}{ll}\eta_{t}^{m} & \eta_{t}^{z}\end{array}\right]^{\prime}$ where $\left\{\eta_{t}^{z}\right\}$ and $\left\{\eta_{t}^{m}\right\}$ denote, respectively, the sequence of technology and non-technology shocks. Following Gali (1999), we identify the technology innovation as the only shock that can have a permanent effect on productivity. This long-run identification imposes a lower triangular structure to $\Theta(I) A_{0}$ that allows the identification of the technology shock.

Let us first provide some intuition about how our "agnostic" method works by discussing what our method would deliver at long horizons. As in Pesavento and Rossi (2003), we use a local-to-unity asymptotic theory to improve the asymptotic approximation to highly persistent processes in small samples. That is, we model the largest root associated to hours, $\rho$, as local-to-unity:

$$
\rho=1+\frac{1}{T} c
$$

To obtain better asymptotic approximations to IRFs in small samples, we also assume that the lead time of the impulse response function, $h$, is a fixed fraction of the sample size:

$$
\frac{h}{T} \underset{T \rightarrow \infty}{\rightarrow} \delta
$$


Note that, because of assumption (5), the method works very well at horizons $(h)$ that are large relative to the available sample size, which is what we refer to as "long horizons".

Considering the two assumptions (4) and (5) together, we have that:

$$
\rho^{h} \underset{T \rightarrow \infty}{\rightarrow} e^{c \delta}
$$

Pesavento and Rossi (2003) show that the IRF of the effect of a technology shock, $\eta_{t}^{z}$, on $n_{t}$ can be approximated by

$$
\frac{\partial n_{t+h}}{\partial \eta_{t}^{z}} \simeq e^{c \delta} \mathbf{i}_{1}^{\prime} \Theta(1) A_{0} \mathbf{i}_{2}
$$

where $\mathbf{i}_{s}$ denotes the $s-t h$ column of the $m \times m$ identity matrix. This provides a simple, closed-form formula for the IRFs at long horizons as a monotone increasing function of $c$. This formula can easily be used to construct confidence intervals for the IRF at long horizons. ${ }^{1}$

To construct IRFs that are valid at short horizons as well, which is what we do in this paper, the method is implemented in practice as follows. (i) We construct a confidence interval for $c$ (denoted by $\left.\left(c_{L} ; c_{U}\right)\right)$ by inverting the acceptance region of a unit root test for hours. One can potentially use any unit root test; in this paper, we use the Augmented Dickey Fuller (ADF) test and Hansen's (1995) CADF test. In our

\footnotetext{
${ }^{1}$ Simply use (6) to obtain the confidence interval as follows: $\left(e^{c_{L} \delta} \mathbf{i}_{1}^{\prime} \widehat{\Theta}(1) \widehat{A}_{0} \mathbf{i}_{2} ; e^{c_{U} \delta} \mathbf{i}_{1}^{\prime} \widehat{\Theta}(1) \widehat{A}_{0} \mathbf{i}_{2}\right)$, where "hats" denote estimated values.
} 
case, the estimated ADF test statistic is -2.068 . Thus, directly from Stock's (1991)

Table A1, p. 455-6, inverting the ADF test delivers a confidence interval for $c$ equal to $(-13.73,2.411)$. (ii) We run a VAR in quasi differences, $(I-\hat{\Phi} L) w_{t}{ }^{2}$ to estimate $\Theta(L)$, and construct a $95 \%$ confidence interval for $\Theta(L) A_{0}$ by using a standard Monte Carlo simulation method (see Hamilton (1994) and Lutkepohl (1993) for details), where $A_{0}$ has been identified as above. More in detail, the confidence interval for $\Theta(L) A_{0}$ is obtained by simulating a confidence interval for $\Theta(L)$, and for every value belonging to the confidence interval we estimate $A_{0}$ that satisfies the identification restriction, which we then use to obtain a confidence interval for $\Theta(L) A_{0}$. (iii) For every horizon, we calculate a confidence interval for $\left[i_{1}^{\prime} \Theta(L) A_{0} i_{2}^{\prime}\right]$ at the relevant horizon, call it $\left(L_{h}, U_{h}\right)$. For example, at horizon $h=1$ this confidence interval is $(-0.435,0.096)$. (iv) Finally, the Bonferroni confidence interval for the response of hours to a technology shock is $\left(e^{c_{L} \delta} L_{h}, e^{c_{U} \delta} U_{h}\right)$. In the example for $h=1$, since $e^{c_{L} \delta}=0.881$ and $e^{c_{U} \delta}=1.023$, we have that the confidence interval for the IRF is $(-0.383,0.098) .{ }^{3}$ While confidence bands constructed in this way have good coverage

\footnotetext{
${ }^{2}$ The quasi-differences are obtained by taking the residuals of a VAR(1). In our empirical application, $\widehat{\Phi}=\left(\begin{array}{cc}0.986 & 0.002 \\ -0.009 & 0.995\end{array}\right)$. As pointed out by a referee, since the estimated value of $\rho(0.986)$ is very close to one, quasi-differencing gives very similar results to first differencing at short horizons.

${ }^{3}$ The last two steps are equivalent (by monotonicity) to the following proceedure. For a given horizon $h=[\delta T]$, for each point on a grid within the confidence interval for $\Theta_{i} A_{0}$, construct two new sequences by multiplying each of the points in the confidence intervals by $e^{c_{L} \delta}$ and $e^{c_{U} \delta}$ respectively, call these sequences $e^{c_{L} \delta} \Theta_{i} A_{0}$ and $e^{c_{U} \delta} \Theta_{i} A_{0}$. The overall confidence interval for the IRF of hours to a productivity shock at horizon $h$ is then obtained as the minimum over the first sequence and the maximum over the second sequence: (min $e^{c_{L} \delta} \mathbf{i}_{1}^{\prime} \Theta_{i} A_{0} \mathbf{i}_{2} ; \max \mathbf{i}_{1}^{\prime} e^{c_{U} \delta} \Theta_{i} A_{0} \mathbf{i}_{2}$ ). By the Bonferroni inequality, the confidence interval should have a coverage of at least $90 \%$ at each horizon $h$. Because exponential functions are always positive, this procedure gives the same result as the procedure described in the main text. Intuitively, relative to simply using (6) with a consistent estimate of $\Theta(I)$ as described in a previous note, step (ii) adds information on the sampling variability of the
} 
properties at short horizons and are robust to the presence of a root close to unity, this comes at the cost of being pointwise and conservative (see Pesavento and Rossi $(2003))^{4}$

In the empirical section we also report results by using Wright's (2000) method. The latter method is implemented by steps (i)-(iv) above, but replacing step (ii) with the following (ii'): $\Theta(L) A_{0}$ is re-estimated conditional on every value of $c$ within a grid over $\left(c_{L}, c_{U}\right)$ - not only at the extremes, like we do. According to Pesavento and Rossi (2003), asymptotically the estimate of $\Theta(L)$ is consistent anyway, and we gain in computational simplicity and smaller confidence bands. In our empirical section, we also report IRFs obtained from standard VAR using $n_{t}$ both in levels and first differences. To estimate the confidence bands in both VARs, we simulate the IRF distribution under a normality assumption with 1000 Monte Carlo replications.

\section{EMPIRICAL RESULTS}

We use the same data as in Christiano et al. (2003), where per capita hours are measured as the natural logarithm of hours worked in the business sector divided by a measure of the working population. Productivity is measured as the natural logarithm of output per hour in the business sector. Data are quarterly observations

short run parameters, $\Theta(L)$, thus improving the performance of the method at short horizons.

${ }^{4}$ Pesavento and Rossi (2003) investigate a variety of methods, all of which have good coverage. These methods build on the inversion of the following test statistics: ADF as in Stock (1991), Elliott, Rothemberg and Stock (1995), Elliott and Stock (2001), Elliott and Jansson (2001) and Elliott, Jansson and Pesavento (2003). While we report results based on ADF and CADF only, our results are qualitatively robust to the use of the other methods mentioned above. 
from 1948:1 to 2001:4 and are ultimately taken from the DRI Economics Database. ${ }^{5}$ As in the previous literature, we identify innovations to technology as the only shocks that have a permanent effect on the level of labor productivity. Figures 1 and 2 report the $90 \%$ confidence intervals and the estimated responses of per capita hours to a one standard deviation positive shock to productivity by using either a VAR in differences or a VAR in levels. ${ }^{6}$ Results from the VAR estimated in differences (Figure 1) are very similar to the results in Gali' (1999) and Francis and Ramey (2001): hours worked show a negative and persistent response to a technology shock in the short run. According to point estimates, the negative effect persists for one year (4 quarters). Eventually, the effect becomes positive in the long run (although not significantly different from zero). When the VAR is estimated by using hours in levels, our results indicate that the initial response of hours is positive, although not significantly different from zero. The response is positive and statistically significant after one quarter, and for roughly twenty quarters.

\section{INSERT FIGURES 1 AND 2}

\footnotetext{
${ }^{5}$ The mnemonics for business labor productivity, business hours and the civilian population over the age of 16 are, respectively: LBOUT, LBMN and P16. We thank Christiano et al. for the data.

${ }^{6}$ The IRF are multiplied by 100 so a value of 0.10 correspond to a response of $0.10 \%$. Following the cited literature, we include a constant, but not a time trend. We focus on a bivariate VAR with hour worked and the productivity measure. As in Francis and Ramey (2001) and in Christiano et al. (2003), we do not expect our results to change if we include additional variables. We use 4 lags (chosen by the BIC criterion) in order to compare our results directly to Francis and Ramey (2001) and Christiano et al. (2003). Results are robust to different lags (e.g. 1 to 6) if we use quasi-differences to estimate the short-run dynamics.
} 
Table 1 shows that indeed hours are a persistent process. The table provides both results on unit root tests on hours and empirical evidence on the magnitude of the persistence by using various methods to construct confidence intervals for the largest root. The methods are: Stock (1991) median unbiased method, Elliott, Rothemberg and Stock (1996), Elliott and Jansson (2001), and Hansen (1995). Stock (1991) method is implemented as follows: first, we calculate the Augmented Dickey Fuller (ADF) test statistic for the time series process of hours with 4 lags; then, by using the "inversion" Table A1, p. 455-6 in Stock (1991), we recover the confidence interval for the largest root. Confidence intervals for the other methods are obtained in a similar fashion, although in the latter cases the inversion table may depend on nuisance parameters and, thus, needs to be calculated by the researcher for the specific database.

According to Stock (1991) method, the largest root is between 0.93 and 1.01, with a median estimate equal to 0.98 . With such a persistent process is not surprising that almost all the tests are not able to reject a unit root at $5 \%$ level (note that CADF test rejects at $10 \%)$. 
Table 1: Unit root tests on per capita hours.

\begin{tabular}{|c|c|c|c|c|}
\hline & Unit root & $5 \%$ & Largest & Root \\
\hline & Test statistic & Critical value & Median estimate & $95 \%$ Confidence interval \\
\hline ADF test & -2.068 & -2.88 & 0.977 & $(0.929 ; 1.015)$ \\
\hline PT test & 23.192 & 3.17 & 1.003 & $(0.988 ; 1.022)$ \\
\hline EJ test & 16.857 & 3.34 & 0.999 & $(0.982 ; 1.021)$ \\
\hline CADF test & -2.437 & -2.65 & 0.971 & $(0.925 ; 1.007)$ \\
\hline $\mathrm{CADF}^{*}$ test & -3.072 & -2.54 & 0.951 & $(0.897 ; 0.997)$ \\
\hline
\end{tabular}

Unit root tests are as follows: "ADF" is the Augmented Dickey Fuller t-test; "PT" is Elliott, Rothemberg and Stock (1996) test. "EJ" and "CADF" are, respectively, Elliott and Jansson (2003) and Hansen (1995) tests, that use information on the stationary covariate, the first difference of productivity. The ADF, PT and EJ tests are implemented with 4 lags, whereas the CADF test is implemented both with 4 lags and 4 leads (CADF) and with 4 lags $\left(\mathrm{CADF}^{*}\right)$. Note that all tests reject when the test statistic is smaller than the critical value.

Given that unit root tests do not strongly support the presence of a unit root, it may not be desirable to take a stand on whether the process has a unit root or not. Kilian and Chang (2000) and Pesavento and Rossi (2003) show that, in the presence of large roots, the coverage rates of confidence intervals for impulse response functions constructed from VARs in first differences or levels can be bad in finite samples. The 
intuition is that a model that imposes a root equal to one when one of the variables is not $\mathrm{I}(1)$ is mis-specified. On the other hand, in small samples, a model in levels underestimates the largest root and the persistence of shocks. These apparently small mistakes and biases become extremely important at medium to long horizons, where the difference between stationary and non-stationary processes becomes more and more important. As a result, VARs in levels and first differences have a very small probability of containing the true impulse response function, almost zero. Unit root pretests do not solve the problem, as the actual coverage of impulse response bands obtained after a pretest can be quite different from the nominal one (due to the low power of unit root tests against persistent alternatives). Furthermore, even if the tests reject a unit root, asymptotic approximations that rely on highly persistent regressors are expected to provide better approximations in small samples. Thus, we use Pesavento and Rossi (2003) "agnostic method" to estimate median unbiased impulse response functions and their confidence bands, which does not require the researcher to choose between the two specifications. By using the local to unity parametrization, we model the persistency of the process as a function of the location parameter $c$ (see the previous section for details), which measures how close to unity the largest root of the process is.

Figure 3 reports results for the "agnostic method". It shows a negative and very short-lived impact effect, which is very much in accordance with the findings of Francis and Ramey (2001). The negative effect lasts only two quarters, less than 
in Francis and Ramey (2001), and it is significant on impact. At business cycle frequencies, the median point estimate of the impulse responses is positive, although not significantly different from zero. The confidence bands show that the effect is very likely to be positive at long horizons and at business cycle frequencies (between 6 quarters and 8 years). Comparing our median unbiased estimate of the response with that of VARs in differences, we find some evidence that the medium and long horizon effect is more positive and slightly larger in magnitude. On the other hand, the effect that we estimate is also more persistent than that obtained from VARs in levels. Finally, for comparison, Figure 4 reports results obtained by using Wrightstyle (2000) methods. ${ }^{7}$ The results are similar, except that the confidence bands are larger. We also checked the robustness of the results to the use of Hansen's (1995) unit root test, which exploits information on stationary covariates, and we find very similar results (see Figure 5). ${ }^{8}$

\section{INSERT FIGURES 3 TO 5}

Our results are also similar to those obtained by using Anderson and Rubin (1949) robust confidence intervals and reported in Vigfusson (2004), p. 11-12. In fact, Vigfusson (2004) finds that, in a bivariate VAR estimated in levels, the impact response

\footnotetext{
${ }^{7}$ The method originally proposed by Wright (2000) is univariate. We apply a method which is in spirit very much similar to his, but it is extended to a multivariate VAR with one large root.

${ }^{8}$ In unreported simulations, we found that the results for the Hansen's (1995) test are robust to whether the CADF test is estimated with both leads and lags (as in Figure 5) or with lags only. We also found that the results are robust to the use of other methods to construct confidence intervals for a unit root, like the Elliott, Rothemberg and Stock (1996) PT test and the Elliott and Jansson (2003) test.
} 
of hours to a one-standard deviation shock can be negative (the confidence interval is $(-0.05,0.11)$ percent $)$ and becomes more positive at business cycle frequencies (the confidence interval is $(0.05,0.27)$ percent after six quarters). In the present paper (see our Figure 3), the "agnostic" estimation shows an impact effect that is negative, but the upper bound of the confidence interval is very close to zero; in addition, after 5-6 quarters the confidence interval becomes more shifted towards positive values, which is very much in line with what Vigfusson (2004) finds.

\section{Conclusions}

This paper analyzed the robustness of the estimate of the effect of a positive productivity shock on hours worked to the presence of a possible unit root in hours. While the literature focused on the cases in which hours are estimated either in levels or in first differences (a sort of "atheist" view), we rely on an "agnostic" procedure in which the researcher does not have to choose between the two specifications. We found that a positive productivity shock has a negative impact effect on hours, as in Francis and Ramey (2001), but the effect is much more short-lived than previously found, and disappears after only two quarters. The effect then becomes positive at business cycle

frequencies, as in Christiano et al. (2003), although it is not significantly different from zero.

Our empirical evidence extends the results in Christiano et al. (2003) in an important and crucial way. In their framework, the level specification implies that the 
first difference specification is mis-specified while the difference specification implies that the level specification is correctly specified. The latter follows from the fact that the level VAR allows for a unit root. While this is true at very short horizons, this does not need to hold at horizons that are large relative to the sample size, where the possibly downward biased estimate of the root becomes important. The importance of these biases depends on the economic problem at hand and on the particular parameters that the researcher faces. Our results show that neglecting this effect may lead to very different economic results in measuring the effects of productivity shocks.

Possible alternative estimation methods include Bayesian methods, as described in Sims and Uhlig (1991). While we do not attempt to pursue this approach in the present paper, a thorough investigation of the performance of Bayesian methods in constructing confidence bands for impulse responses is provided in Kilian and Chang (2000). 


\section{Figure 1. Estimation in differences}

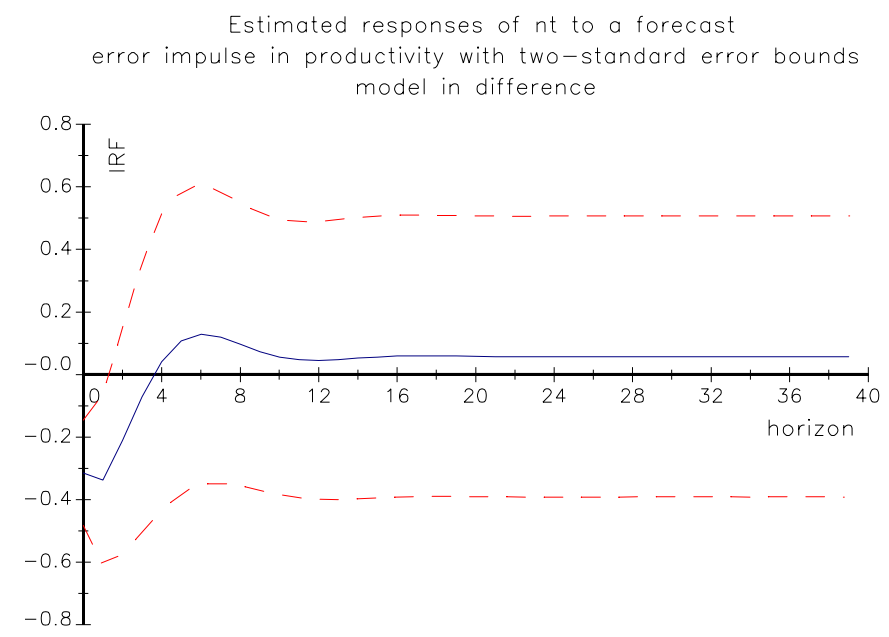

Figure 2. Estimation in levels

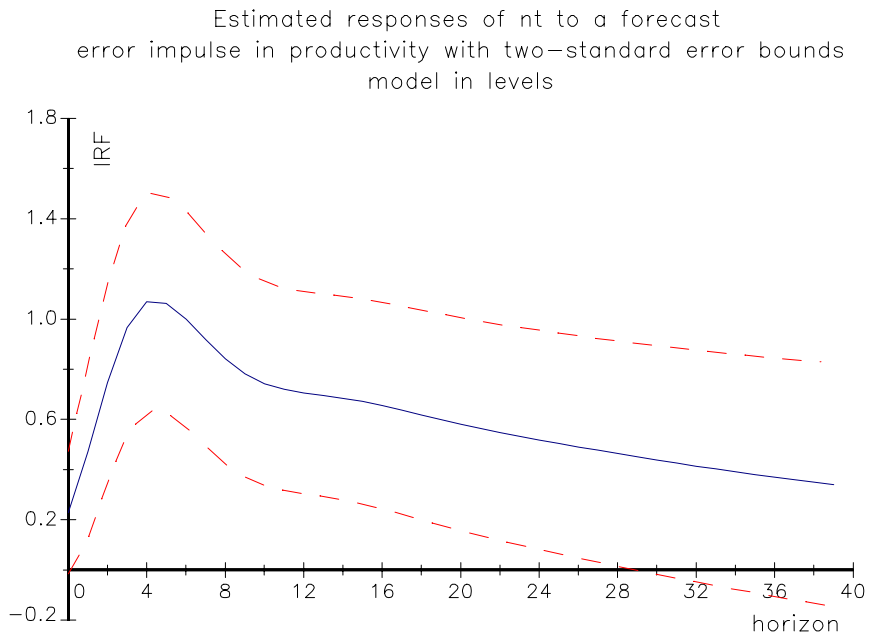

Figures 1 and 2 show the estimated IRF (solid line) and IRF confidence bands (dotted line) of hours worked to a one percent standard deviation increase in the productivity shock. The model is a VAR in differences in Figure 1 and in levels in Figure 2 . 


\section{Figure 3. Agnostic estimation}

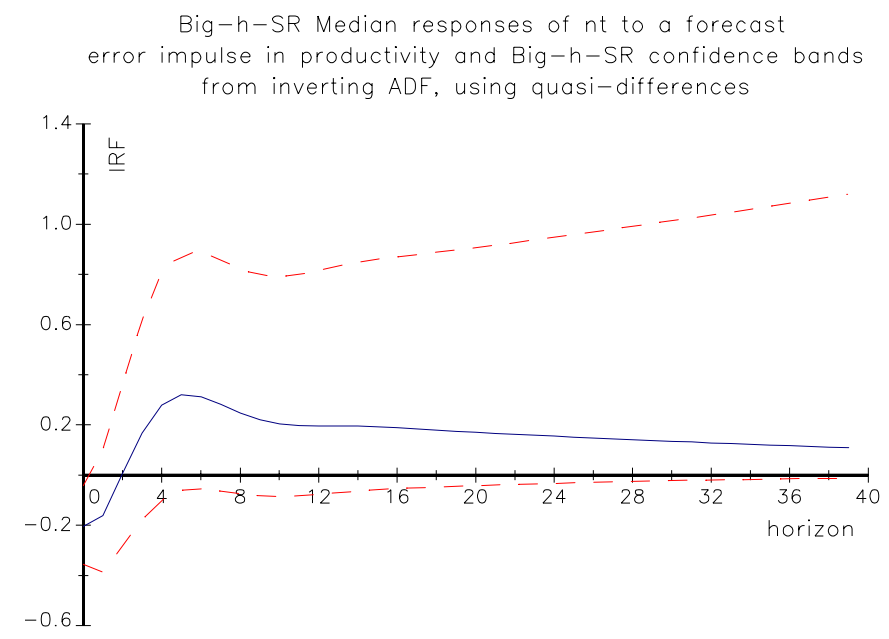

Figure 4. Wright method

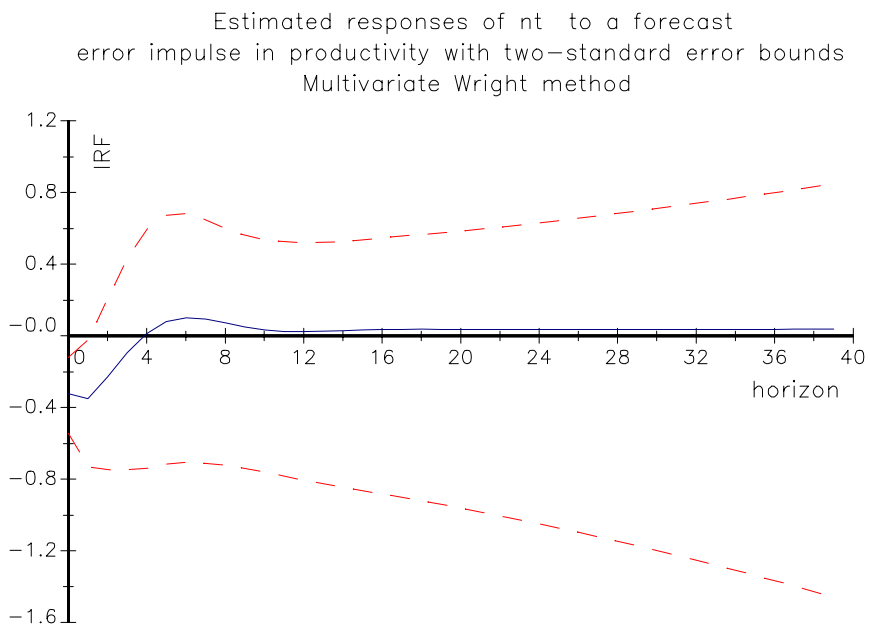

Figures 3 shows the estimated IRF confidence bands of hours worked to a one percent standard deviation increase in the productivity shock. Results based on Pesavento and Rossi (2003) method robust at short horizons. Figure 4 shows the IRF estimate and the confidence bands that we obtain by applying a method similar to Wright (2000). 


\section{Figure 5. Agnostic estimation CADF}

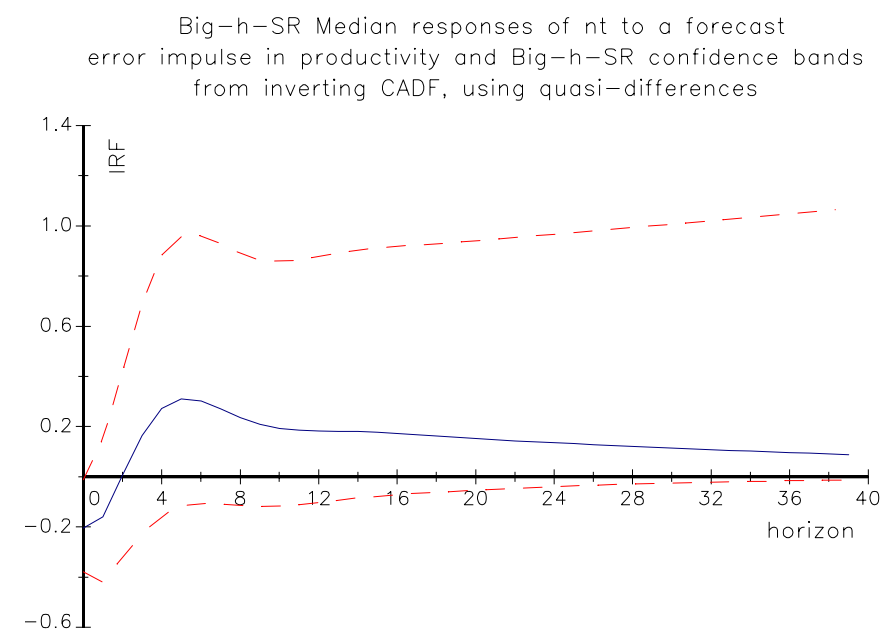

Figure 5 shows the IRF estimate and the confidence bands that we obtain by applying a method based on Hansen (1995) test. 


\section{REFERENCES}

Anderson, T.W. and H. Rubin (1949), "Estimation of the Parameters of A Single Equation in A Complete System of Stochastic Equations", Annals of Mathematical Statistics 20, pp. 46-63.

Christiano, L., M. Eichenbaum and R. Vigfusson (2003), "What Happens After a Technology Shock?",

Board of Governors of the Federal Reserve System, International Finance Discussion Paper, 768.

Elliott, G. and M. Jansson (2003), "Testing for Unit Roots with Stationary Covariates", Journal of Econometrics, 115, pp. 75-89.

Elliott, G., M. Jansson and E. Pesavento (2002), "Optimal Power for Testing Potential Cointegrating Vectors with Known Parameters for Nonstationarity", Journal of Business and Economic Statistics, forthcoming.

Elliott, G., Thomas J. Rothenberg and J. H. Stock (1996), "Efficient Tests for an Autoregressive Unit Root", Econometrica, 64, pp. 813-836.

Elliott, G. and J. H. Stock (2001), "Confidence Intervals for Autoregressive Coefficients Near One", Journal of Econometrics, 103, pp. 155-181.

Francis, N., M. Owyang and A. Theodorou (2003), "The Use of Long-Run Restrictions for the Identification of Technology Shocks", Federal Reserve Bank of St. 
Louis Review, 85(6), 53-66.

Francis, N. and V. Ramey (2001), "Is the Technology-Driven Real Business Cycle Hypothesis Dead? Shocks and Aggregate Fluctuations Revisited", Journal of Monetary Economics, forthcoming.

Gali, J. (1999), "Technology, Employment, and the Business Cycle: Do Technology Shocks Explain Aggregate Fluctuations?", American Economic Review, 89(1), 249-271.

Gali, J. and P. Rabanal (2004), “Technology Shocks and Aggregate Fluctuations: How Well Does the RBC Model Fit Postwar U.S. Data?", in: B. Bernanke and K. Rogoff, eds., NBER Macroeconomic Annual, MIT Press.

Hamilton, James D., Time Series Analysis, Princeton University Press, 1994.

Hansen, B. (1995), "Rethinking the Univariate Approach to Unit Root Testing: Using Covariates to Increase Power", Econometric theory 11(5), 1148-71.

Kilian, L. and P. Chang (2000), "How accurate are confidence intervals for impulse responses in large VAR models?", Economics Letters 69, pp. 299-307.

Lütkepohl, Helmut, Introduction to Multiple Time Series Analysis, Second Edition. New York: Springer-Verlag, 1993. 
Pesavento, E. and B. Rossi (2003), "Small Sample Confidence Intervals for Multivariate Impulse Response Functions at Long Horizons", Duke University Working Paper \#03-19.

Shea, J. (1999), "What Do Technology Shocks Do?", in B. Bernanke and J. Rotemberg, eds., NBER Macroeconomics Annual, Cambridge, MA: MIT Press.

Sims, C. and H. Uhlig (1991), "Understanding Unit Rooters: A Helicopter Tour", Econometrica 59(6), 1591-99.

Stock, J. H. (1991), "Confidence Intervals for the Largest Autoregressive Root in U.S. Macroeconomic Time Series", Journal of Monetary Economics, 28, pp. 435-459.

Uhlig, H. (2003), "Do Technology Shocks Lead to a Fall in Total Hours Worked?", mimeo.

Vigfusson, R. (2004), “The Delayed Response to A Technology Shock: A Flexible Price Explanation", Board of Governors of the Federal Reserve System, International Finance Discussion Paper, 810, July 2004.

Wright, J. (2000), "Confidence Intervals for Univariate Impulse Responses with a Near Unit Root", Journal of Business and Economic Statistics 18(3). 